\title{
Brazilian waxing and human papillomavirus: a case of acquired epidermodysplasia verruciformis
}

\author{
Mark G. Kirchhof MD PhD, Sheila Au MD
}

Competing interests: Sheila Au has received funds from La RochePosay, Ono Pharmaceutical Co., Valeant

Pharmaceuticals International Inc., Amgen, AbbVie, Galderma, Valeo Pharma and Janssen. No competing interests were declared by Mark Kirchhof.

This article has been peer reviewed.

The authors have obtained patient consent.

\section{Correspondence to:} Mark Kirchhof, kirchhof .mark@gmail.com

CMAJ 2015. DOI:10.1503 /cmaj.140198
A 48-year-old woman presented with traumatic injury secondary to removal of pubic hair using hot wax. Her medical history included long-standing type 1 diabetes mellitus, complicated by retinopathy, nephropathy and neuropathy. Because of diabetic complications, she had required a renal transplant five years before presentation and a pancreatic transplant two years later. Her antirejection medications included tacrolimus, mycophenolate mofetil and prednisone. Her past dermatologic history included melasma on the face.

Three years after her pancreatic transplant, the patient underwent "Brazilian" waxing. When the wax was pulled off the skin, the entire area was denuded and bleeding. The patient was assessed by a physician, and the traumatized area was allowed to heal by secondary intention. As the area healed, the patient noticed a violaceous discoloration in her pubic area and again sought medical attention. On examination, a purplish-red plaque over the mons pubis extending to the inguinal folds was observed. At the border of this plaque, brownish-red planar verrucous papules were noted, with sparing of the posterior aspects of the vulva, buttocks and perineal area (Figure 1). A biopsy was taken and sent for histopathology.

Histopathology showed keratinocytes with changes consistent with the effects of human papillomavirus (HPV), and a diagnosis of epidermodysplasia verruciformis was made. She reported no personal or family history of this condition. We initiated a side-to-side comparison of treatment with liquid nitrogen cryotherapy on the right upper medial thigh and inguinal crease, and tretinoin $0.1 \%$ cream daily on the left side. On follow-up, the side treated with liquid nitrogen showed much more postinflammatory hyperpigmentation and less resolution of lesions, and the side treated with tretinoin showed more satisfactory flattening of the papules and minimal postinflammatory hyperpigmentation. There was some erythema and discomfort with the tretinoin treatment, but these adverse effects diminished with time.

\section{Discussion}

The issues raised in this case represent the intersection of several trends that are becoming increasingly important to physicians. First, there is a rise in the number of patients who are immunosuppressed. ${ }^{1}$ Not only is the number of solid organ transplant recipients growing, but so too is the number of patients taking immunosuppressive medication for a variety of inflammatory and autoimmune disorders. The second trend involves the increasing popularity of body modification, including partial or complete removal of body hair. One recent survey-based study found that more than $70 \%$ of teenage girls shaved or waxed their pubic hair on a regular basis. ${ }^{2}$ This predilection for hairlessness is also increasing among men. The methods used to remove body hair also include shaving, clipping, epilation, depilatory creams, laser hair removal, electrolysis, sugaring and threading. Many of these methods cause trauma that can provide an entry point for infection.

Because of a lack of controlled trials on the risks and adverse effects of techniques for body hair removal, case reports provide most of our insight into the risks of body hair removal. One report discussed a patient with diabetes mellitus in whom life-threatening Streptococcus pyogenes and herpes simplex virus infections developed after Brazilian waxing. ${ }^{3}$ In another case 
report, an outbreak of methicillin-resistant Staphylococcus aureus was linked to cosmetic body shaving. ${ }^{4}$ Two cases of HPV infection linked to threading have been reported. ${ }^{5}$ These patients had facial hair removed by threading, and subsequently verrucae developed at the sites of hair removal. Furthermore, HPV has been shown to reside on pubic and perianal hairs. ${ }^{6}$ It follows that traumatic hair removal in these areas might result in an infection and subsequent clinical manifestation of HPV. In the case of our patient, HPV-associated epidermodysplasia verruciformis developed, secondary to pubic hair removal.

\section{Epidermodysplasia verruciformis}

There are two described subtypes of epidermodysplasia verruciformis, the classic type and the acquired type. Classic epidermodysplasia verruciformis is an autosomal recessive genodermatosis that increases susceptibility to particular HPV subtypes. ${ }^{7}$ The HPV subtypes that are commonly associated with epidermodysplasia verruciformis include HPV $-5,-8,-9,-12,-14,-15,-17,-19$, $-25,-36,-38,-47$ and $-50 .{ }^{8}$ These HPV subtypes are also found on the skin of people without epidermodysplasia verruciformis; however, they are rarely pathogenic in immunocompetent individuals. ${ }^{9}$ Although the actual pathogenesis for the development of epidermodysplasia verruciformis has not been completely elucidated, it is widely hypothesized to be associated with defects in cell-mediated immunity.

Classic epidermodysplasia verruciformis begins in early childhood with flat wart-like papules and plaques on the extremities, neck, face and trunk. There is a high association with the development of squamous cell carcinoma, particularly in sun-exposed areas. ${ }^{9}$ Cutaneous malignancy will develop in about half of these patients by the fourth or fifth decade. ${ }^{9}$

Recently, an acquired form of epidermodysplasia verruciformis has been described in immunocompromised patients. ${ }^{7}$ Most of these patients are infected with HIV, but transplant patients taking antirejection drugs and patients with hematologic malignancy taking antineoplastic medications are also susceptible. ${ }^{7}$ The risk of malignant transformation secondary to acquired epidermodysplasia verruciformis is unknown, but clinical surveillance with appropriate follow-up is warranted given the risks associated with inherited forms of epidermodysplasia verruciformis.

As with many other HPV infections of the skin, epidermodysplasia verruciformis is associated with epithelial hyperplasia. Topical or systemic retinoids, which have an antiprolifer- ative effect, can be used to control the growth of the epidermis. In addition, retinoids may provide the added benefit of slowing or preventing the progression to dysplasia and malignancy. ${ }^{10}$ In a few cases, systemic retinoids were combined with interferon, which provided an even better clinical response. ${ }^{8}$ In patients with classic epidermodysplasia verruciformis, imiquimod has been used topically with clinical improvement. ${ }^{8}$ Imiquimod is an immune response modulator that activates the immune system through toll-like receptor 7 (TLR-7) and is used commonly for treatment of verrucae and condylomata. Imiquimod is also used in the treatment of Bowen disease or squamous cell carcinoma in situ and as such may provide further benefit in treating dysplastic lesions or early malignancies. Topical cidofovir and topical podophyllotoxin have also been used, but the clinical results are not as reliable or substantial as those achieved with imiquimod or retinoids. ${ }^{7,8}$ Surgical approaches can also be used, including cryotherapy, electrodessication and curettage, as well as surgical excision.

Acquired epidermodysplasia verruciformis is more difficult to treat than the classic form. ${ }^{7}$ Topical imiquimod, topical 5-fluorouracil and isotretinoin have been tried in this patient group without success. ${ }^{7}$ Given the lack of evidence available regarding the ideal first-line treatment for acquired epidermodysplasia verruciformis, we engaged in a side-to-side com-

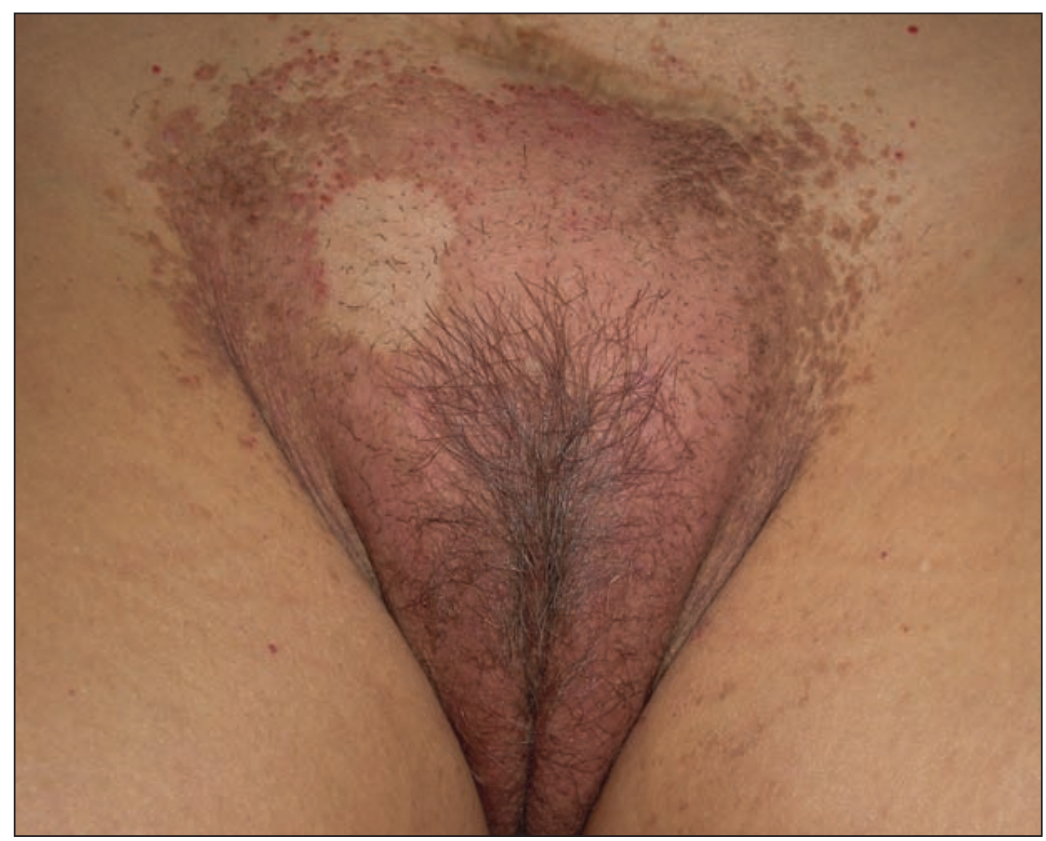

Figure 1: Clinical photograph of the groin of a 48-year-old woman showing purplish-red flat-topped verrucous papules coalescing into a confluent plaque over the mons pubis and labia. These flat wart-like lesions developed after pubic hair waxing. 
parison of topical versus destructive therapy to assess the best course of action for our patient. Changing the immune status by putting patients with HIV on effective antiretroviral therapy has had mixed results. ${ }^{8}$ Consideration can be given to altering the dose or type of antirejection medications in the transplant population, but the risks of rejection must be weighed against the unproven clinical effects on epidermodysplasia verruciformis.

\section{Conclusion}

We have presented a case of acquired epidermodysplasia verruciformis in a patient with immunosuppression whose infection was precipitated by pubic hair removal using hot wax. The growing demographic of patients who are immunosuppressed and the increasing prevalence of pubic hair removal suggest that cases such as this one may be seen more often in physicians' offices. Hair removal techniques that minimize trauma to the epidermis, such as trimming or use of depilatory creams, should be discussed with patients who are immunosuppressed.

\section{References}

1. Sung RS, Galloway J, Tuttle-Newhall JE, et al. Organ donation and utilization in the United States, 1997-2006. Am J Transplant 2008;8:922-34.

2. Bercaw-Pratt JL, Santos XM, Sanchez J, et al. The incidence, attitudes and practices of the removal of pubic hair as a body modification. J Pediatr Adolesc Gynecol 2012;25:12-4.

3. Dendle C, Mulvey S, Pyrlis F, et al. Severe complications of a "Brazilian" bikini wax. Clin Infect Dis 2007;45:e29-31.

4. Begier EM, Frenette K, Barrett NL, et al. A high-morbidity outbreak of methicillin-resistant Staphylococcus aureus among players on a college football team, facilitated by cosmetic body shaving and turf burns. Clin Infect Dis 2004;39:1446-53.

5. Kumar R, Zawar V. Threading warts: a beauty parlor dermatosis. J Cosmet Dermatol 2007;6:279-82.

6. Poljak M, Kocjan BJ, Potocnik M, et al. Anogenital hairs are an important reservoir of alpha-papillomaviruses in patients with genital warts. J Infect Dis 2009;199:1270-4.

7. Rogers HD, Macgregor JL, Nord KM, et al. Acquired epidermodysplasia verruciformis. J Am Acad Dermatol 2009;60:315-20.

8. Gewirtzman A, Bartlett B, Tyring S. Epidermodysplasia verruciformis and human papilloma virus. Curr Opin Infect Dis 2008;21: 141-6.

9. Patel T, Morrison LK, Rady P, et al. Epidermodysplasia verruciformis and susceptibility to HPV. Dis Markers 2010;29:199-206.

10. Marquez C, Bair SM, Smithberger E, et al. Systemic retinoids for chemoprevention of non-melanoma skin cancer in high-risk patients. J Drugs Dermatol 2010;9:753-8.

Affiliations: Department of Dermatology and Skin Science (Kirchhof, Au), University of British Columbia, Vancouver, BC; Division of Dermatology, Department of Medicine (Au), St. Paul's Hospital, Vancouver, BC

Contributors: Both authors drafted and revised the article, and approved the final version submitted for publication.

\section{How you can get involved in the CMA!}

The CMA is committed to providing leadership for physicians and promoting the highest standard of health and health care for Canadians. To strengthen the association and be truly representative of all Canadian physicians the CMA needs to hear from members interested in serving in elected positions and on appointed committees and advisory groups. The CMA structure comprises both governing bodies and advisory bodies either elected by General Council or appointed by the CMA Board of Directors. The Board of Directors - elected by General Council — has provincial/territorial, resident and student representation, is responsible for the overall operation of the CMA and reports to General Council on issues of governance.

CMA committees advise the Board of Directors and make recommendations on specific issues of concern to physicians and the public. Five core committees mainly consist of regional, resident and student representation while other statutory and special committees and task forces consist of individuals with interest and expertise in subject-specific fields. Positions on one or more of these committees may become available in the coming year.

For further information on how you can get involved please go to https://www.cma.ca/en/Pages/get-involved-in-cma.aspx, or contact

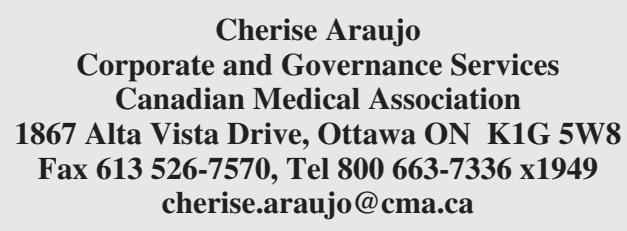

By getting involved, you will have an opportunity to make a difference.

We hope to hear from you!

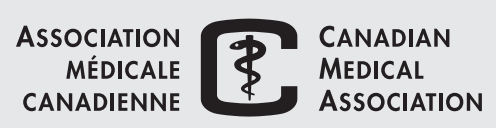

